\title{
Novel approximate distribution of the sum of lognormal-Rician turbulence channels with pointing errors and applications in MIMO FSO links
}

This paper was downloaded from TechRxiv (https://www.techrxiv.org).

\section{LICENSE}

CC BY 4.0

SUBMISSION DATE / POSTED DATE

03-09-2021 / 10-09-2021

\section{CITATION}

$\mathrm{Li}$, Xiaofeng; miao, maoke (2021): Novel approximate distribution of the sum of lognormal-Rician turbulence channels with pointing errors and applications in MIMO FSO links. TechRxiv. Preprint.

https://doi.org/10.36227/techrxiv.16566192.v1

$\mathrm{DOI}$

10.36227/techrxiv.16566192.v1 


\title{
Novel approximate distribution of the sum of lognormal-Rician turbulence channels with pointing errors and applications in MIMO FSO links
}

\author{
Maoke Miao ${ }^{\mathrm{a}}$, Xiaofeng $\mathrm{Li}^{\mathrm{a}, *}$ \\ ${ }^{a}$ School of Aeronautics and Astronautics, University of Electronic Science and Technology \\ of China, Chengdu, China
}

\begin{abstract}
In this paper, a novel approximate closed-form probability density function expression for the sum of lognormal-Rician turbulence channels with Rayleigh pointing errors is developed. The results of Kolmogorov-Smirnov goodness-offit statistical tests show that the proposed approximation is highly accurate across a wide range of channel conditions. Also, the analysis of approximation error is presented in detail, and it indicates that a more efficient approximation can be achieved for larger coherence parameter $r$ and smaller variance $\sigma_{z}^{2}$. To reveal the importance of proposed approximation, new approximate closed-form expressions of the ergodic capacity, outage probability, and bit-error rate are derived. The performance for multiple-input multiple-output (MIMO) free-space optical (FSO) systems with equal gain combining (EGC) diversity technique are analyzed with different parameters, including the number of transmit and receive apertures, turbulence channels, and presence of pointing errors. The asymptotic ergodic capacity at high signal-to-noise ratio is also obtained and shown to be closer to the simulation results when compared to the other methods. It is observed that MIMO technology can offer a significant improvement in system performance. Numerical results demonstrate the accuracy of the proposed approach.
\end{abstract}

\footnotetext{
${ }^{*}$ Corresponding author

Email address: lxf3203433@uestc.edu.cn (Xiaofeng Li)
}

Preprint submitted to Elsevier

September 3, 2021 
Keywords: lognormal-Rician turbulence channels, Rayleigh pointing errors, Kolmogorov-Smirnov statistical tests, MIMO FSO communication systems, EGC diversity.

\section{Introduction}

Free-space optical (FSO) communication, also known as outdoor optical wireless communication, has attracted considerable attention in recent years owing to its attractive bandwidth enhancement with unregulated spectrum. Furthermore, it can be anticipated that the FSO systems are likely to be noteworthy in the development of fifth-generation $(5 \mathrm{G})$ as they efficiently overcome the important challenge caused by the radio frequency (RF) spectrum shortage [1. Despite these benefits of FSO technology, its widespread use has been hampered by its disappointing performance for long range links due to atmospheric turbulence-induced fading and pointing errors [2].

Plenty of statistical models have been proposed to characterize the turbulenceinduced scintillation in FSO systems. The lognormal distribution is generally accepted for weak turbulence conditions [3, 4]. The Gamma-Gamma distribution is a double-stochastic scintillation model, which is shown to provide an excellent agreement with the experimental data in the moderate-to-strong turbulence regime [5]. However, it is shown in [6] that this model only performs well for receivers with small aperture. Another important distribution is the lognormal-Rician distribution (also known as Beckmann), which comprises the lognormal, lognormal-Exponential and exponential distribution as its special cases [7. The lognormal-Rician distribution not only agrees well with experimental data under weak-to-strong turbulence regime, but also it is more accurate than the Gamma-Gamma distribution under a spherical wave assumption in particular [5, 8]. In recent years, a new universal turbulence model, named Malaga distribution has been widely advocated as it generalizes several turbulence-induced fading models (including Gamma-Gamma, lognormal) 9]. To apply these turbulence models to the analyses of practical FSO systems, 
we are often required to estimate the corresponding unknown parameters [10]. However, the PDF of Malaga distribution is expressed with a finite summation and includes plenty of parameters, making parameter estimation more difficult for parameter estimation when compared to Gamma-Gamma and lognormalRician channels. Also, it should be noted that the lognormal distribution is not an exact relation to the Malaga distribution, implying that lognormal-Rician is an approximate case of the Malaga distribution [11.

Apart from the turbulence-induced fading, the performance of FSO systems can be also severely deteriorated by the pointing errors caused by building sway or thermal expansions [12. Various types of statistical models have been developed to describe the properties of pointing errors. The pioneer study of the pointing errors model in FSO systems can be found in [13, which considered the effects of beam width, detector size and independent identical Gaussian distributions for the elevation and the horizontal displacement. Note that Rayleigh pointing errors model have been popularly used in a great deal of research articles due to its simplicity from a mathematical point of view as well as due to its realistic approach [14 16].

Multiple-input multiple output (MIMO) technology has emerged as a promising technology to mitigate the effects of scintillation and pointing errors effects. Over the last couple of decades, a great amount of work has been conducted to study the performance of MIMO FSO links over different turbulence channels with pointing errors. For instance, the authors in [15 17] dealt with the ergodic capacity, bit-error rate (BER) and outage probability performance over Gamma-Gamma fading with pointing errors. The work in [18 analyzed the BER performance of MIMO FSO links employing the maximal ratio combining (MRC) technique over the Malaga fading with pointing errors while the secrecy outage probability and secrecy capacity performance were investigated in [19]. With respect to the lognormal-Rician model, the results available so far for single-input single-output (SISO) and MIMO FSO systems in the literature are greatly limited since it does not has the closed-form PDF expression compared to the Gamma-Gamma and Malaga distribution. In [20, the author introduced 
the saddlepoint approximation method to approximate the lognormal-Rician distribution. In [8], the authors investigated the BER performance of coherent FSO systems in lognormal-Rician turbulence employing the MRC and select combining (SC) via the Padé approximation method. Also, the authors in [21] employed the Gram-Charlier Series to study the BER performance of MIMO FSO systems with MRC. However, it must be noted that these three kinds of numerical method provides limited information for performance analysis, i.e., the impacts of channel parameters on the systems performance can not be calculated. Moreover, based on the method introduced in [22], asymptotic ergodic capacity at high signal-to-noise ratio (SNR) regime as well as at low SNR regime for SISO FSO links with Rayleigh pointing errors were developed in 23. Nevertheless, this method can not be used to derive the ergodic capacity at medium SNR.

Based on aforementioned discussion, we investigate the performance of MIMO FSO systems over lognormal-Rician turbulence channels with pointing errors in this work. To this end, we first develop a new statistical formula to approximate the PDF of composite channels by using a series representation. The Kolmogorov-Smirnov (KS) goodness-of-fit statistical tests results indicate that proposed approximation is highly accurate over a wide range of channel conditions. In order to reveal the importance of the proposed statistical formulation, the ergodic capacity, outage probability, and BER performance for MIMO FSO systems with EGC diversity technique are studied. The asymptotic ergodic capacity at high SNR is also derived to provide further insights. To the best of our knowledge, all the obtained expressions are new in the open literature. Monte Carlo simulation results confirm that the obtained approximate analytic results are highly efficient. 


\section{System and channel model}

\subsection{System model}

Let us consider an intensity modulation and direct detection (IMDD)-based MIMO FSO systems with $M_{t}$ transmitters and $N_{r}$ receivers, where an OOK symbol $x \in\{0,1\}$ is transmitted by all transmit apertures for each transmission time interval. Note that the IMDD systems are commonly used in the terrestrial FSO links due to their simplicity and low cost of implementation. Additionally, the photodetectors are spatially separated by a sufficient distance so that the channels are considered to be statistically independent and uncorrelated 24, 25]. Moreover, assuming that a large amount of background radiation is collected by each receiver, and this justifies the use of the additive white Gaussian noise (AWGN) model as a good approximation of the Poisson photon counting detection model 24]. Hence, the received signal at the $j$ th, $j=1, \cdots, N_{r}$ receive aperture is given as

$$
r_{j}=\eta \sum_{i=1}^{M_{t}} I_{i, j} x+v_{j}
$$

where $\eta$ is the optical-to-electrical conversion coefficient, $v_{j}$ is AWGN with zero

mean and variance of $\sigma^{2}=N_{0} / 2$, i.e., $v_{j} \sim \mathcal{N}\left(0, \frac{N_{0}}{2}\right) . I_{i, j}$ denotes the fading channel coefficient between the $i$ th transmit aperture and $j$ th receive aperture, and is subjected to the combined effects of turbulence-induced irradiance fluctuation and pointing errors. Here, the EGC diversity technique is employed at the receiver as it provides a performance close to that of the MRC while having the advantage of implementation simplicity [26]. The signal at the output of the receiver is expressed as

$$
r=\eta \sum_{i=1}^{M_{t}} \sum_{j=1}^{N_{r}} I_{i, j} x+\sum_{j=1}^{N_{r}} v_{j} .
$$

The corresponding electrical SNR of the combined signal is given by

$$
\gamma_{E G C}=\frac{\eta^{2} \mathbb{E}\left[|x|^{2}\right]\left(\sum_{i=1}^{M_{t}} \sum_{j=1}^{N_{r}} I_{i, j}\right)^{2}}{N_{r} \sigma^{2}}=\frac{\gamma_{0} I_{T}^{2}}{N_{r}}
$$


where $\gamma_{0}=\eta^{2} \mathbb{E}\left[|x|^{2}\right] / \sigma^{2}$ denotes the average SNR and $I_{T}=\sum_{i=1}^{M_{t}} \sum_{j=1}^{N_{r}} I_{i, j}$.

\subsection{Channel model}

The irradiance $I_{i, j}$ is considered to be the product of atmospheric turbulenceinduced scintillation $I_{i, j}^{a}$ and pointing errors $I_{i, j}^{p}$, i.e., $I_{i, j}=I_{i, j}^{a} I_{i, j}^{p}$. As in [12, these two fading factors are approximately independent for smaller jitter values. To characterize the fading $I_{i, j}^{a}$ over a wide range of turbulence conditions, the lognormal-Rician distribution model is adopted here as it provides an excellent fit with both simulation and experimental data in weak-to-strong turbulence regimes. The probability density function $(\mathrm{PDF})$ of $I_{i, j}^{a}$ is given by $[7$.

$$
\begin{gathered}
f_{I_{i, j}^{a}}\left(h_{a}\right)=\frac{(1+r) \exp (-r)}{\sqrt{2 \pi} \sigma_{z}} \int_{0}^{\infty} \frac{1}{z^{2}} I_{0}\left(2 \sqrt{\frac{h_{a} r(1+r)}{z}}\right) \\
\exp \left[-\frac{1+r}{z}-\frac{1}{2 \sigma_{z}^{2}}\left(\log (z)+\frac{1}{2} \sigma_{z}^{2}\right)^{2}\right] d z
\end{gathered}
$$

where $r \geq 0$ is the coherence parameter that represents the ratio of total power in the dominant (LOS) components to that of scattered components, $\sigma_{z}^{2}$ is the variance of the logarithm of the irradiance modulation factor $z$, and $I_{0}$ is the zero-order modified Bessel function of the first kind [27. Note that Eq. (4) evolves from the product of a Rician amplitude and a lognormal modulation factor, i.e., $I_{i, j}^{p}=z y$. The PDFs of them are respectively expressed as

$$
\begin{aligned}
& f_{Z}(z)=\frac{1}{\sqrt{2 \pi} \sigma_{z} z} \exp \left[-\frac{1}{2}\left(\frac{\log (z)+\frac{1}{2} \sigma_{z}^{2}}{\sigma_{z}}\right)^{2}\right] \\
& f_{Y}(y)=(1+r) \exp (-r-(1+r) I) I_{0}(2 \sqrt{r(1+r) I}) .
\end{aligned}
$$

From Eq. (5), it is easy to see the lognormal-Rician distribution comprises some well-known models as its special cases according to the different combinations of shape parameters. For example, the lognormal-Rician distribution reduces to the lognormal distribution as $r$ approaches infinity. Also, it specialized to the lognormally modulated exponential distribution when $r$ approaches 0 .

Another performance limiting factor in FSO links is the pointing errors, which arise due to misalignment between the transmitter and receiver. It is 
noted that such effects are likely to occur in urban areas in particular, where FSO equipment is placed on high-rise structures [28, 29]. According to [13, the attenuation due to geometric spread and pointing errors can be approximated as

$$
I_{i, j}^{p}(r) \approx A_{0} \exp \left(-2 \frac{r^{2}}{w_{z e q}^{2}}\right), r \geq 0
$$

where $A_{0}=[\operatorname{erf}(v)]^{2}$ is the fraction of the collected power at $r=0, v=$ $\sqrt{\pi} R_{a} / \sqrt{2} w_{z}, R_{a}$ is the receiver's aperture radius, and $w_{z}$ is the beam waist that can be approximated by $w_{z}=\theta z$ with $\theta$ denoting the transmit divergence angle. $w_{z e q}=\sqrt{w_{z}^{2} \sqrt{\pi} \operatorname{erf}(v) / 2 v \exp \left(-v^{2}\right)}$ represents the equivalent beam waist. It must be mentioned that the approximation in Eq. (5) provides a good agreement with the exact value when the normalized beam width $w_{z} / R_{a}>6$. For convenience, the independent identical Gaussian distributions for the elevation and the horizontal displacement (sway) are assumed, being $\sigma_{s}^{2}$ the jitter variance at the receiver. Thus, the PDF of radical displacement $r$ follows a Rayleigh distribution, which is defined as $[13$.

$$
f(r)=\frac{r}{\sigma_{s}^{2}} \exp \left(-\frac{r^{2}}{2 \sigma_{s}^{2}}\right) .
$$

Combining Eqs. (6) and (7), the probability distribution of $I_{i, j}^{p}$ becomes

$$
f\left(I_{i, j}^{p}\right)=\frac{\xi^{2}}{A_{0}^{\gamma^{2}}}\left(I_{i, j}^{p}\right)^{\xi^{2}-1}, 0 \leq I_{i, j}^{p} \leq A_{0}
$$

where $\xi=w_{z e q} / 2 \sigma_{s}$.

\section{An efficient approximation to the distribution of $I_{T}$}

\subsection{Approximate distribution for the sum of $L$ random variates}

Based on the discussion of the previous section, we firstly deal with the challenges of the distribution of the sum of lognormal-Rician random variables (RVs) with pointing errors. For convenience, these channels are assumed to be statistically independent and identically distributed (i.i.d). The proposed methodology shown in the following has been partly introduced in [16, 30, 
where the distribution of the sum of independent Gamma-Gamma variates with (without) pointing errors are investigated.

Let $\left\{I_{l}\right\}_{l=1}^{L}$ be a set of $L$ i.i.d RVs that follows a lognormal-Rician with pointing errors distribution. Their sum is defined as

$$
S=\sum_{l=1}^{L} I_{l}=\sum_{l=1}^{L} W_{l} u\left(r_{l}\right)=\sum_{l=1}^{L} z_{l} y_{l} u\left(r_{l}\right)
$$

where $W_{l}=z_{l} y_{l}$, and $z_{l}, y_{l}, u\left(r_{l}\right)$, and $r_{l}$ are lognormal, Rician, pointing errors, and Rayleigh RVs respectively. Using a similar methodology presented in [30, Eq. (9)], Eq. (9) can be rewritten as

$$
\begin{aligned}
S= & \frac{\sum_{l=1}^{L} z_{l} \sum_{l=1}^{L} y_{l} \sum_{l=1}^{L} u\left(r_{l}\right)}{L^{2}}+\frac{1}{L^{2}} \sum_{i=1}^{L-1} \sum_{j=i+1}^{L}\left(z_{i}-z_{j}\right)\left(y_{i}-y_{j}\right) \sum_{l=1}^{L} u\left(r_{l}\right) \\
& +\frac{1}{L} \sum_{i=1}^{L-1} \sum_{j=i+1}^{L}\left(z_{i} y_{i}-z_{j} y_{j}\right)\left(u\left(r_{i}\right)-u\left(r_{j}\right)\right) .
\end{aligned}
$$

Hence, according to Eq. 10, the unknown distribution $S$ can be approximated by the distribution of the RV $\tilde{S}$, which is defined as the first term in Eq. 10, namely

$$
S \approx \tilde{S}=\frac{\sum_{l=1}^{L} z_{l} \sum_{l=1}^{L} y_{l} \sum_{l=1}^{L} u\left(r_{l}\right)}{L^{2}}
$$

Hence, the summation of second and third terms in Eq. 10 represent the approximation error $\epsilon$, which is given by

$$
\begin{aligned}
\epsilon= & \epsilon_{1}+\epsilon_{2} \\
= & \frac{1}{L^{2}} \sum_{i=1}^{L-1} \sum_{j=i+1}^{L}\left(z_{i}-z_{j}\right)\left(y_{i}-y_{j}\right) \sum_{l=1}^{L} u\left(r_{l}\right) \\
& +\frac{1}{L} \sum_{i=1}^{L-1} \sum_{j=i+1}^{L}\left(z_{i} y_{i}-z_{j} y_{j}\right)\left(u\left(r_{i}\right)-u\left(r_{j}\right)\right) .
\end{aligned}
$$

It can be easily seen the $\epsilon$ goes to 0 for $L=1$. Next, we are now solving the

distribution for RV $\tilde{S}$. Note that the sum of i.i.d lognormal variates can be efficiently approximated by an another lognormal distribution, as in [31]. Thus, 
the $\mathrm{PDF}$ of $\mathrm{RV} G=\sum_{l=1}^{L} z_{l}$ is derived as

$$
f_{G}(g)=\frac{1}{\sqrt{2 \pi \sigma_{L}^{2}} g} \exp \left(-\frac{\left(\log (g)-\mu_{L}\right)^{2}}{2 \sigma_{L}^{2}}\right)
$$

where the parameters $\mu_{L}, \sigma_{L}^{2}$ are respectively given by [31]

$$
\begin{aligned}
\sigma_{L}^{2} & =\log \left(\frac{\left(\exp \left(\sigma_{z}^{2}\right)-1\right)}{L}+1\right) \\
\mu_{L} & =\log (L)-\frac{\sigma_{L}^{2}}{2} .
\end{aligned}
$$

Specifically, according to [32, TableIV], the series representation for Eq. 13 is expressed as

$$
f_{G}(g) \approx \frac{1}{\sqrt{\pi}} \sum_{k=1}^{K} \frac{w_{k}}{u_{k}} \mathbf{H}_{0,0}^{0,0}\left[\left.\frac{g}{u_{k}}\right|_{-} ^{-}\right]
$$

where $\mathbf{H}_{p, q}^{m, n}\left(\left.\lambda\right|_{\left(b_{j}, B_{j}\right)_{j=1: q}} ^{\left(a_{j}, A_{j}\right)_{j=1: p}}\right)$ is the Fox's H function [33], $u_{k}=\exp \left(\sqrt{2} \sigma_{L} \omega_{k}+\mu_{L}\right)$, $w_{k}$ and $\omega_{k}$ are the weight factor and zeros (abscissas) of the $K$-order Hermite polynomial [34]. The moment generating function (MGF) of $f_{G}(g)$ becomes [32, 35]

$$
M_{G}(s)=\frac{1}{\sqrt{\pi}} \sum_{k=1}^{K} w_{k} \exp \left(-u_{k} s\right)
$$

It is shown in [36] that the squared Rician distribution is actually a special case of $\kappa-\mu$ distribution with $\kappa=r, \mu=1, \Omega=1$. Hence, invoked by the [37, which demonstrates that the sum of L i.i.d $\kappa-\mu \mathrm{RVs}$ with parameters $\kappa=r, \mu, \Omega$ is also a $\kappa-\mu \mathrm{RV}$ with parameters $\kappa=r, L \mu, L \Omega$. Then, the PDF of RV $\mathrm{V}=$ $\sum_{l=1}^{L} y_{l}$ becomes

$$
f_{V}(v)=\frac{L(1+r)^{\frac{L+1}{2}} v^{\frac{L-1}{2}}}{\exp (L r) r^{\frac{L-1}{2}} L^{\frac{L+1}{2}}} \exp (-(1+r) v) I_{L-1}(2 \sqrt{r(1+r) v L}) .
$$

The above formula Eq. (17) can be rewritten as

$$
f_{V}(v)=\sum_{q=0}^{Q} \frac{(r L)^{q}}{q ! \Gamma(L+q) v}((1+r) v)^{L+q} \exp (-r L-(1+r) v)
$$


where we have applied the series expansion result onto the $I_{L-1}$ [27, Eq (8.445)]. Alternatively, Eq. 18 is equivalent to

$$
\begin{aligned}
f_{V}(v) & =\sum_{q=0}^{Q} \frac{\exp (-r L)(r L)^{q}}{l ! \Gamma(L+q)}(1+r)^{L+q} v^{L+q-1} \mathbf{H}_{0,1}^{1,0}\left[\left.(1+r) v\right|_{0,1} ^{-}\right] \\
& =\sum_{q=0}^{Q} \frac{\exp (-r L)(r L)^{q}}{q ! \Gamma(L+q)}(1+r) \mathbf{H}_{0,1}^{1,0}\left[\left.(1+r) v\right|_{L+q-1,1} ^{-}\right]
\end{aligned}
$$

where in Eq. 19, we have expressed the exp $(\cdot)$ in terms of the Fox's H function [38, Eq. (2.5)] and the second equality in Eq. (19) holds due to [38, Eq. (2.4)]. Hence, combining Eqs. (15) and (19), the probability distribution for the RV $T_{1}=\frac{1}{L} V G$ is obtained as

$$
f_{T_{1}}\left(t_{1}\right) \approx \frac{1}{\sqrt{\pi}} \sum_{q=0}^{Q} \sum_{k=1}^{K} \frac{w_{k}}{u_{k}} \frac{L \exp (-r L)(r L)^{q}}{q ! \Gamma(L+q)}(1+r) \mathbf{H}_{0,1}^{1,0}\left[\left.\frac{(1+r) t_{1} L}{u_{k}}\right|_{(L+q-1,1)} ^{-}\right]
$$

where we have used the [38, Theorem. (4.1)] in the above formula. Moreover, Eq. 201 can be further reduced to

$$
f_{T_{1}}\left(t_{1}\right) \approx \frac{1}{\sqrt{\pi}} \sum_{q=0}^{Q} \sum_{k=1}^{K} w_{k} \frac{\exp (-r L)(r L)^{q}}{q ! \Gamma(L+q)}\left(\frac{(1+r) L}{u_{k}}\right)^{L+q} t_{1}^{L+q-1} \exp \left(-\left(\frac{1+r}{u_{k}}\right) t_{1} L\right)
$$

after using the following relation [39, Eq. (1.125)]

$$
\mathbf{H}_{0,1}^{1,0}\left[\left.z\right|_{(b, B)} ^{-}\right]=B^{-1} z^{\frac{b}{B}} \exp \left(-z^{\frac{1}{B}}\right) .
$$

Additionally, $T_{2}=\frac{1}{L} \sum_{l=1}^{L} u\left(r_{l}\right) \approx F u\left(\frac{1}{L} \sum_{l=1}^{L} r_{l}^{2}\right)$, and its PDF can be efficiently approximated by [16]

$$
f_{T_{2}}\left(t_{2}\right) \approx(-1)^{L-1} \frac{L^{L} g^{L}}{t_{2} 2^{L} \Gamma(L)} \ln ^{L-1}\left(\frac{t_{2}}{F A_{0}}\right)\left(\frac{t_{2}}{F A_{0}}\right)^{\frac{L g}{2}}, 0 \leq t_{2} \leq F A_{0}
$$

where $g=\frac{w_{z e q}^{2}}{2 \sigma_{s}^{2}}=2 \xi^{2}$, and $F=\frac{(2+g L)^{L}}{L^{L} g^{L-1}(2+g)}$ that satisfies $\lim _{g \rightarrow \infty} F=1$. Now, the distribution of $\tilde{S}=T_{1} T_{2}$ is finally approximated by that of $\hat{S}$, which is found to 
be

$$
\begin{aligned}
f_{\tilde{S}}(s) \approx & f_{\hat{S}}(s)=\frac{1}{\sqrt{\pi}} \sum_{q=0}^{Q} \sum_{k=1}^{K} w_{k} \frac{\exp (-r L)(r L)^{q}}{q ! \Gamma(L+q)}\left(\frac{(1+r)}{u_{k} A_{0} F} L\right)^{\frac{L g}{2}} \frac{L^{L} g^{L}}{2^{L}} s^{\frac{L g}{2}-1} \\
& G_{L, L+1}^{L+1,0}\left(\left.\frac{s(1+r)}{u_{k} A_{0} F} L\right|_{L+q-\frac{L g}{2}, 0, \cdots, 0} ^{1, \cdots, 1}\right)
\end{aligned}
$$

where $G_{p, q}^{m, n}(\cdot \mid \cdots)$ denotes the Meijer's G-function [27. A full derivation of Eq. (24) is given in Appendix A. Note that the derived approximate analytic solution of composite channel PDF in Eq. (24) facilitates the performance analysis of FSO systems and has not been obtained in the literature. Also, it should be emphasized that the convergence of these two series are dependent on the channel state and number of apertures. For numerical evaluation the series Eq. (24) is truncated to a finite number of terms. From extensive simulation studies, we find that $Q=50$ and $K=15$ are sufficient to achieve convergence for all of the numerical examples in the paper. Furthermore, by utilizing [40, Eq. (26)], the cumulative density function (CDF) of the combined fading can be approximated as

$$
\begin{aligned}
F_{\hat{S}}(a) \approx & \frac{1}{\sqrt{\pi}} \sum_{q=0}^{Q} \sum_{k=1}^{K} w_{k} \frac{\exp (-r L)(r L)^{q}}{q ! \Gamma(L+q)}\left(\frac{(1+r)}{u_{k} A_{0} F} L\right)^{\frac{L g}{2}} \frac{L^{L} g^{L}}{2^{L}} a^{\frac{L g}{2}} \\
& G_{L+1, L+2}^{L+1,1}\left(\left.\frac{a(1+r)}{u_{k} A_{0} F} L\right|_{L+q-\frac{L g}{2}, 0, \cdots, 0,-\frac{L g}{2}} ^{1-\frac{L g}{2}, 1, \cdots, 1}\right) \\
\approx & \frac{1}{\sqrt{\pi}} \sum_{q=0}^{Q} \sum_{k=1}^{K} w_{k} \frac{\exp (-r L)(r L)^{q}}{q ! \Gamma(L+q)} \frac{L^{L} g^{L}}{2^{L}} \\
& G_{L+1, L+2}^{L+1,1}\left(\left.\frac{a(1+r)}{u_{k} A_{0} F} L\right|_{L+q, L g / 2, \cdots, L g / 2,0} ^{1,1+L g / 2, \cdots, 1+L g / 2}\right)
\end{aligned}
$$

where we have used the following relation [27, Eq. (9.31.5)]

$$
z^{k} G_{p, q}^{m, n}\left(\left.z\right|_{b_{1}, \cdots, b_{q}} ^{a_{1}, \cdots, a_{p}}\right)=G_{p, q}^{m, n}\left(\left.z\right|_{b_{1}+k, \cdots, b_{q}+k} ^{a_{1}+k, \cdots, a_{p}+k}\right) .
$$




\subsection{The approximation error $\epsilon_{t}$ analysis}

Based on the information described above, the main ideas of our proposed approximate method are summarized as follows:

$$
S \approx \tilde{S}=\frac{\sum_{l=1}^{L} z_{l} \sum_{l=1}^{L} y_{l} \sum_{l=1}^{L} u\left(r_{l}\right)}{L^{2}} \approx \hat{S}=\frac{\phi\left(\sum_{l=1}^{L} z_{l}\right) \sum_{l=1}^{L} y_{l} F u\left(\sqrt{\frac{1}{L} \sum_{l=1}^{L} r_{l}^{2}}\right)}{L}
$$

where $\phi(\cdot)$ denotes some function. It should be noted that the value of RV

$\phi\left(\sum_{l=1}^{L} z_{l}\right)$ in each generation extremely close to that of $\left(\sum_{l=1}^{L} z_{l}\right)$, and the PDF expression of $\phi\left(\sum_{l=1}^{L} z_{l}\right)$ is shown in Eq. 13. According to Eq. 27), the corresponding approximation error $\epsilon_{t}$ is given by

$$
\epsilon_{t}=\epsilon+(\tilde{S}-\hat{S})
$$

Next, we make great efforts to analyze the $\epsilon_{t}$. The exact PDF of the error $\epsilon_{t}$ is difficult to be derived, however, its first and second moments, which are indicative of its statistical behavior [30], can be calculated. Hence, the mean of $\epsilon_{t}$ is derived as

$$
\mathbb{E}\left[\epsilon_{t}\right]=\mathbb{E}[\epsilon]+\mathbb{E}(\tilde{S}-\hat{S})
$$

It is easy to see that the mean of $\epsilon$ is equal to 0 since the $z_{l}, y_{l}$, and $u\left(r_{l}\right)$ are statistically independent. The mean of $\tilde{S}$ and $\hat{S}$ are respectively obtained as

$$
\mathbb{E}[\tilde{S}]=L \frac{A_{0} \xi^{2}}{1+\xi^{2}}
$$

and

$$
\begin{aligned}
\mathbb{E}[\hat{S}]=\int_{0}^{\infty} f_{\hat{S}}(s) s d s & =\frac{1}{\sqrt{\pi}} \sum_{q=0}^{Q} \sum_{k=1}^{K} w_{k} \frac{\exp (-r L)(r L)^{q}}{q !}(L+q) \frac{u_{k} A_{0} F}{(1+r) L} \frac{L^{L} g^{L}}{(L g+2)^{L}} \\
& =\frac{1}{\sqrt{\pi}} \sum_{q=0}^{Q} \sum_{k=1}^{K} w_{k} \frac{\exp (-r L)(r L)^{q}}{q !}(L+q) \frac{u_{k} A_{0}}{(1+r) L} \frac{\xi^{2}}{1+\xi^{2}}
\end{aligned}
$$

where in Eq. (30) we have used the second equation in Eq. 61), and in Eq. (31) we have used the integration formula [16, Eq. (81)]. Using that the Taylor series 
expansion of $\exp (x)$ at $x=0$ [27, Eq. (1.211.1)], we have

$$
\sum_{q=0}^{Q} \frac{(r L)^{q}}{q !}(L+q) \approx \exp (r L) L(r+1)
$$

Knowing the fact that the first derivative of $M_{G}(s)$ at $s=0$ is $\mathbb{E}[G]=L$, i.e.

$$
\left.\frac{d M_{G}(s)}{d s}\right|_{s=0}=L=\frac{1}{\sqrt{\pi}} \sum_{k=1}^{K} w_{k} u_{k}
$$

where the second equality holds due to Eq. (16). Then, we find that Eq. (31) reduces to Eq. (30) after substituting Eqs. (32) and (33) into Eq. (31). Therefore, we have

$$
\mathbb{E}\left[\epsilon_{t}\right]=0
$$

The variance of $\epsilon_{t}$ is given by

$$
\mathbb{E}\left[\epsilon_{t}^{2}\right]=\mathbb{E}\left[\epsilon^{2}\right]+\mathbb{E}\left[(\tilde{S}-\hat{S})^{2}\right]
$$

where the variance of $\mathbb{E}\left[\epsilon^{2}\right]$ is obtained as

$$
\begin{aligned}
\mathbb{E}\left[\epsilon^{2}\right] & =\mathbb{E}\left[\epsilon_{1}^{2}\right]+\mathbb{E}\left[\epsilon_{2}^{2}\right]+2 \mathbb{E}\left[\epsilon_{1} \epsilon_{2}\right] \\
& =\mathbb{E}\left[\epsilon_{1}^{2}\right]+\mathbb{E}\left[\epsilon_{2}^{2}\right]
\end{aligned}
$$

The second equation in Eq. 36 holds due to

$$
\mathbb{E}\left[\left(\sum_{l=1}^{L} u\left(r_{l}\right)\right)\left(u\left(r_{i}\right)-u\left(r_{j}\right)\right)\right]=0 .
$$

The variance of $\epsilon_{1}$ and $\epsilon_{2}$ are respectively derived as

$$
\mathbb{E}\left[\varepsilon_{1}^{2}\right]=\frac{(L-1)}{L} \frac{(1+2 r)}{(1+r)^{2}}\left(\exp \left(\sigma_{z}^{2}\right)-1\right)\left(\frac{A_{0}^{2} \xi^{2}}{2+\xi^{2}}+(L-1) \frac{A_{0}^{2} \xi^{4}}{\left(1+\xi^{2}\right)^{2}}\right)
$$

and

$$
\mathbb{E}\left[\varepsilon_{2}^{2}\right]=(L-1)\left(\frac{2+4 r+r^{2}}{(1+r)^{2}} \exp \left(\sigma_{z}^{2}\right)-1\right)\left(\frac{A_{0}^{2} \xi^{2}}{2+\xi^{2}}-\frac{A_{0}^{2} \xi^{4}}{\left(1+\xi^{2}\right)^{2}}\right)
$$

A full derivation of Eqs. (38) and (39) is given in Appendix B. Also, the above two equations indicate that the variance of the approximation error increases 
for a certain combination of $r, \sigma_{z}^{2}, A_{0}$ and $\xi$ as the number of RVs of the sum in Eq. (9) increases. Specifically, we have

Proposition 1: The variance of $\epsilon$ is equal to that of $\epsilon_{1}$ as $\xi$ approaches $\infty$, which is given by

$$
\mathbb{E}\left[\varepsilon^{2}\right]=\mathbb{E}\left[\varepsilon_{1}^{2}\right]=(L-1) \frac{(1+2 r)}{(1+r)^{2}}\left(\exp \left(\sigma_{z}^{2}\right)-1\right) A_{0}^{2} .
$$

Proof: Proposition 1 can be easily proved according to $\lim _{\xi \rightarrow \infty} \mathbb{E}\left[\varepsilon_{2}^{2}\right]=0$.

Corollary 1: A more efficient approximation can be achieved for a larger $\xi$.

Proof: Substituting $A_{0}=\left[\operatorname{erf}\left(\sqrt{\pi} R / \sqrt{2} w_{z}\right)\right]^{2}$ into Eq. 40 and using the relation $d \operatorname{erf}(x) / d x=2 \exp \left(-x^{2}\right) / \sqrt{\pi}$ [27, Eq. (8.250.1)], then the first derivative of $\mathbb{E}\left[\epsilon^{2}\right]$ is strictly less than zero according to Eq. 41 .

$$
\begin{aligned}
\frac{d \mathbb{E}\left[\epsilon^{2}\right]}{d w_{z} / R}= & -(L-1) \frac{(1+2 r)}{(1+r)^{2}}\left(\exp \left(\sigma_{z}^{2}\right)-1\right) 2\left(\operatorname{erf}\left(\sqrt{\pi / 2} /\left(w_{z} / R\right)\right)\right) \\
& \frac{\sqrt{2} \exp \left(-\pi / 2 /\left(w_{z} / R\right)^{2}\right)}{\left(w_{z} / R\right)^{2}}<0
\end{aligned}
$$

The variance of $\mathbb{E}\left[(\tilde{S}-\hat{S})^{2}\right]$ is difficult to be solved since function $\phi(\cdot)$ unknown. However, it can be approximated with high accuracy in the following way

$$
\mathbb{E}\left[(\tilde{S}-\hat{S})^{2}\right] \approx\left(\sum_{l=1}^{L} z_{l} \sum_{l=1}^{L} y_{l}\right)^{2} \mathbb{E}\left[\left(\frac{1}{L^{2}} \sum_{l=1}^{L} u\left(r_{l}\right)-\frac{F}{L} u\left(\sqrt{\frac{1}{L} \sum_{l=1}^{L} r_{l}^{2}}\right)\right)^{2}\right]
$$

In Fig. 1. we investigate the tightness of the Eq. (36) and Eq. (40) by showing the numerical results. As can be seen, the variance of $\epsilon_{1}$ approaches the variance of $\epsilon^{2}$ as $\xi$ increases. Specifically, they nearly overlap with each other for $w_{z} / R_{a}=$ $14, \sigma_{s} / R_{a}=1(\xi=7.02)$, which verifies the argument of Proposition 1. In addition, we find that the variance of $\epsilon$ decreases with increasing value of $w_{z} / R_{a}$ for given $\sigma_{s} / R_{a}$, and this is also shown in Fig. 2, where we presents the Monte Carlo simulation results of $\mathbb{E}\left[(\tilde{S}-\hat{S})^{2}\right]$. By comparing curves in Figs. 11 and 2. we conclude a larger variance of approximation error is obtained when the number of $L$ increases or $w_{z} / R_{a}$ decreases. 


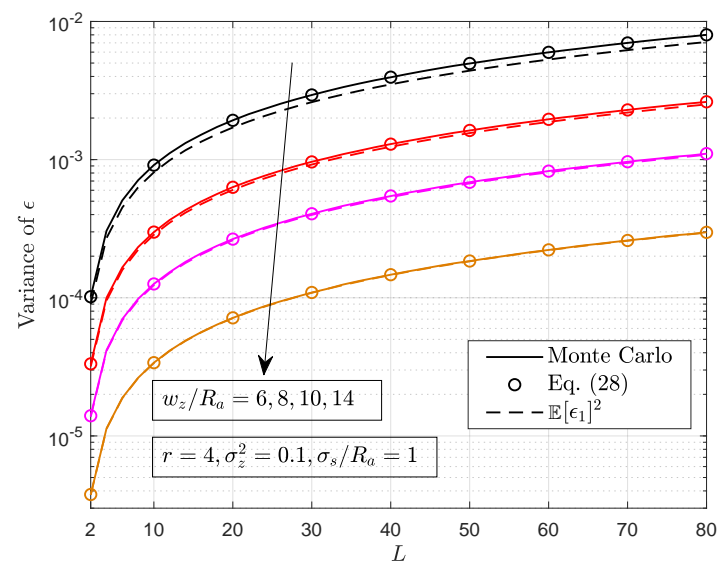

Figure 1: The variance of $\epsilon$ for different pointing errors and $L$.

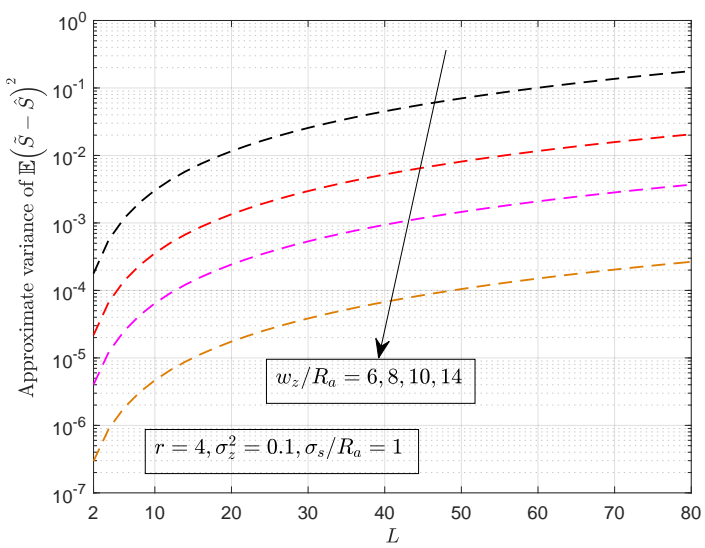

Figure 2: Monte Carlo simulation results of approximate variance $\mathbb{E}\left[(\tilde{S}-\hat{S})^{2}\right]$ for different pointing errors and $L$. 


\subsection{Numerical examples}

In this section, we investigate the accuracy of the proposed approximate PDF expression by comparing the statistic of the simulation data. Note that the simulated PDFs and CDFs generalized from $1 \times 10^{6}$ samples by Monte Carlo method are used for reference. Fig. 3 depicts the PDF and CDF results for different combinations of turbulence conditions, apertures, and pointing errors. It is clearly illustrated that the approximate results always provide an excellent agreement with the simulation results.

To quantitatively evaluate the error behavior of the approximation method, we here employ the KS goodness-of-fit statistical tests tools, which measure the maximum value of absolute difference between the empirical cumulative distribution function $(\mathrm{CDF})$ of the $\mathrm{RV} S, F_{S}(\cdot)$ and the approximate analytical CDF expression $F_{\hat{S}}(\cdot)$ shown in Eq. (25). Hence, the KS test statistic is defined as

$$
T=\max \left|F_{S}(a)-F_{\hat{S}(a)}\right| \cdot
$$

It is shown in [41] that an approximation is considered to be accepted with significance level $1-\alpha$ if the statistical test value $T$ is less than given critical value $T_{\max }$, while it is said to be rejected with the same significance if $T>T_{\max }$.

The critical value $T_{\max }$ is given by $T_{\max }=\sqrt{-\frac{1}{2 N} \log \left(\frac{\alpha}{2}\right)}$ with $\alpha$ denoting the significant level and $N$ is the number of samples. The typical value for them are set to be $\alpha=5 \%$ and $N=10^{4}$ respectively, resulting in the critical value $T_{\max }=0.0136$. In Fig. (4), we study the KS test statistic of the approximation for different combinations of $r, \sigma_{z}^{2}, w_{z} / R_{a}, \sigma_{s} / R_{a}$ and $L$. It must be emphasized that the present results are obtained by averaging the results of 100 simulation runs. According to Fig. 4 we find that the proposed approximation performs better for the larger $r$ or smaller $\sigma_{z}^{2}$. Specifically, it is accepted with $95 \%$ significance in all the range of the parameters examined when $L=2, w_{z} / R_{a}=$ $8, \sigma_{s} / R_{a}=1$. The impact of pointing errors on the approximation accuracy can be concluded by comparing the curves in Fig. 4(a) and Fig. 4(b), which indicates that a more efficient approximation can be achieved for the smaller 

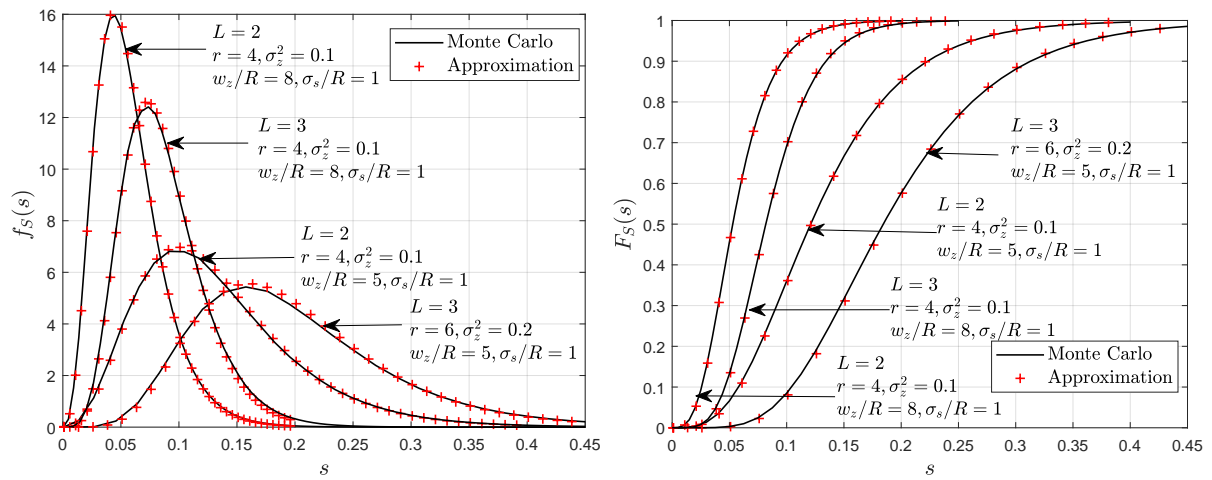

Figure 3: PDF and CDF plots of the Monte Carlo simulation and the approximate distributions for different combinations of turbulence conditions, apertures, and pointing errors.

pointing errors. As expected, the number of apertures $L$ has a negative effect on the approximation accuracy when comparing the statistic test results in Fig. 4 (b) and Fig. 4(c).

\section{Performance analysis for the MIMO FSO systems}

To revel the importance of proposed approximation, we study the ergodic capacity, outage probability and BER performance of MIMO FSO systems over lognormal-Rician fading channels with pointing errors.

\subsection{Ergodic capacity analysis}

Assuming the instantaneous channel side information is perfectly known at the receiver, the ergodic capacity corresponding to the considered MIMO FSO systems in bits $/ \mathrm{Hz} / \mathrm{s}$ is given by

$$
\begin{aligned}
C_{\mathrm{MIMO}, \mathrm{pe}} & =\int_{0}^{\infty} \log _{2}\left(1+\frac{\gamma_{0}}{N_{r}} s^{2}\right) f_{S}(s) d s=\int_{0}^{\infty} \frac{1}{\log (2)} G_{2,2}^{1,2}\left(\left.\gamma_{0} s^{2}\right|_{1,0} ^{1,1}\right) f_{S}(s) d s \\
& \approx \hat{C}_{\mathrm{MIMO}, \mathrm{pe}}=\int_{0}^{\infty} \frac{1}{\log (2)} G_{2,2}^{1,2}\left(\left.\gamma_{0} s^{2}\right|_{1,0} ^{1,1}\right) f_{\hat{S}}(s) d s
\end{aligned}
$$

where in Eq. 444), we have expressed $\log (1+a x)$ in terms of Meijer's G-function [27, Eq. (8.4.6.5)]. Substituting Eq. (24) into Eq. (44) and using the formula 


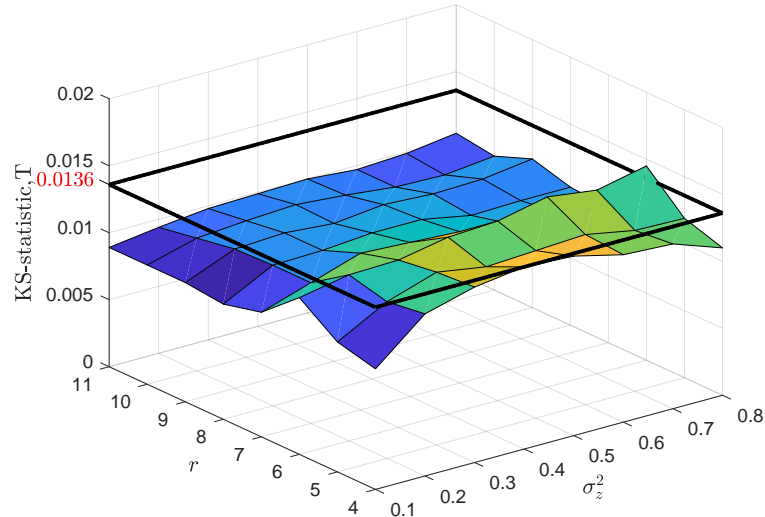

(a) $L=2, w_{z} / R_{a}=5, \sigma_{s} / R_{a}=1$.

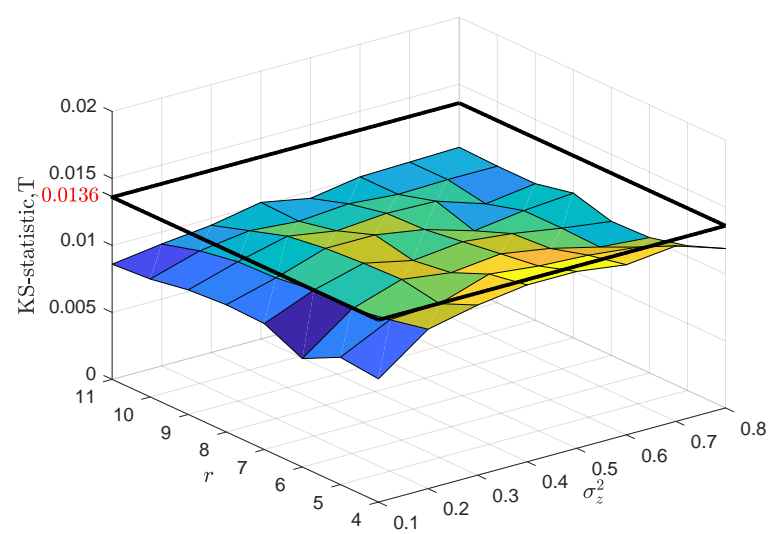

(b) $L=2, w_{z} / R_{a}=8, \sigma_{s} / R_{a}=1$.

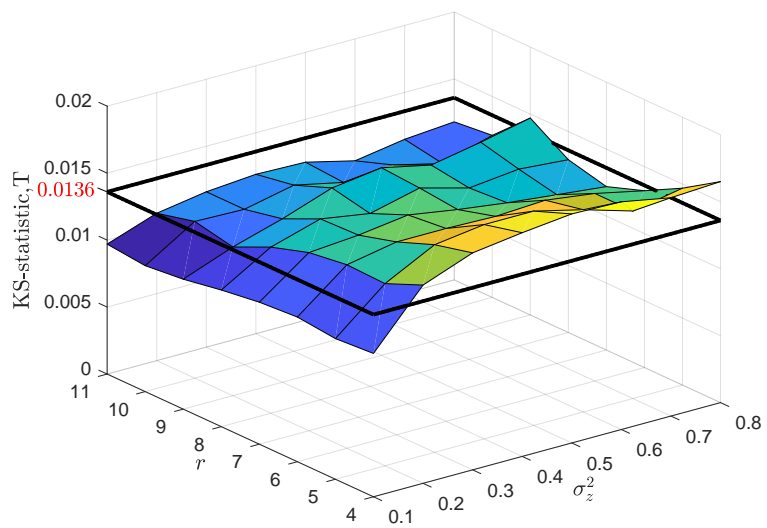

(c) $L=3, w_{z} / R_{a}=8, \sigma_{s} / R_{a}=1$.

Figure 4: KS goodness-of-fit tests results for different combinations of turbulence conditions, apertures, and pointing errors. 
[42, the approximate closed-form expression for the ergodic capacity of MIMO FSO communication system is obtained as

$$
\begin{aligned}
& C_{\mathrm{MIMO}, \mathrm{pe}} \approx \frac{\log _{2}(\exp (1))}{\pi} \sum_{q=0}^{Q} \sum_{k=1}^{K} w_{k} \frac{\exp (-r L)(r L)^{q}}{q ! \Gamma(L+q)} \frac{L^{L} g^{L}}{2^{L}} \\
& 2^{q-1} G_{2 L+4,2 L+2}^{1,2 L+4}\left(\left.\frac{4 \frac{\gamma_{0}}{N_{r}}}{\left(\frac{(1+r)}{u_{k} A_{0} F} L\right)^{2}}\right|_{1,0,\left(\frac{1-\frac{L g}{2}-1}{2}, \frac{2-\frac{L g}{2}-1}{2}\right)^{\otimes L}} ^{1,1, \frac{1-L-q}{2}, \frac{2-L-q}{2},\left(\frac{1-\frac{L g}{2}}{2}, \frac{2-\frac{L g}{2}}{2}\right)^{\otimes L}}\right) \\
& \approx \frac{\log _{2}(\exp (1))}{\pi} \sum_{q=0}^{Q} \sum_{k=1}^{K} w_{k} \frac{\exp (-r L)(r L)^{q}}{q ! \Gamma(L+q)} \frac{L^{L} g^{L}}{2^{L}} \\
& 2^{q-1} G_{2 L+4,2 L+2}^{1,2 L+4}\left(\left.\frac{4 \frac{\gamma_{0}}{N_{r}}}{\left(\frac{(1+r)}{u_{k} A_{0} F} L\right)^{2}}\right|_{1,0,\left(\frac{1-\frac{L g}{2}-1}{2}\right)^{\otimes L}} ^{1,1, \frac{1-L-q}{2}, \frac{2-L-q}{2},\left(\frac{2-\frac{L g}{2}}{2}\right)^{\otimes L}}\right)
\end{aligned}
$$

where $(\cdot)^{\otimes L}$ denotes the $L$ times repetition of a given tuple and the second equality in Eq. 45 due to [27, Eq. (9.301)]. It can be see that the approximate expression involves the Meijer's G-function, and thus does not provide much insight on the capacity performance. Also, we are always interested in the ergodic capacity in the high SNR regime. Note that $\log _{2}\left(1+\gamma_{0} / N_{r} s^{2}\right)$ can be approximated by $\log _{2}\left(\gamma_{0} / N_{r} s^{2}\right)$ in this case. As such, we have

$C_{\mathrm{MIMO}, \mathrm{pe}, \mathrm{hsnr}} \approx \int_{0}^{\infty} \log _{2}\left(\gamma_{0} / N_{r} s^{2}\right) f_{S}(s) d s \approx \log _{2}\left(\gamma_{0} / N_{r}\right)+\int_{0}^{\infty} 2 \log _{2}(s) f_{\hat{S}}(s) d s$. 
Substituting Eq. (24) into Eq. (46), we can obtain an asymptotic expression of ergodic capacity at high SNRs as

$$
\begin{aligned}
C_{\mathrm{MIMO}, \mathrm{pe}, \mathrm{hsnr}} \approx & \log _{2}(\exp (1))\left(\frac{1}{\sqrt{\pi}} \sum_{q=0}^{Q} \sum_{k=1}^{K} w_{k} \frac{\exp (-r L)(r L)^{q}}{q !}\right. \\
& \left.\left(-\frac{4}{g}+2 \varphi(0, L+q)+2 \ln \left(\frac{u_{k} A_{0} F}{(1+r) L}\right)\right)+\ln \left(\frac{\gamma_{0}}{N_{r}}\right)\right) \\
\approx & \log _{2}(\exp (1))\left(2 \sum_{q=0}^{Q} \frac{\exp (-r L)(t L)^{q}}{q !} \varphi(0, L+q)\right. \\
& \left.-\frac{4}{g}-\sigma_{L}^{2}+2 \log \left(\frac{A_{0} F}{(1+r)}\right)+\log \left(\frac{\gamma_{0}}{N_{r}}\right)\right)
\end{aligned}
$$

where $\varphi(\cdot)$ is the digamma function [27, Eq. (8.365.4)] and we have used the following integration formula [16, Eq. (83)] in deriving the first equality of Eq. (47)

$\int_{0}^{\infty} t^{q_{1}-1} \log (t) G_{L, L+1}^{L+1,0}\left(\left.t\right|_{q_{2}, 0, \cdots, 0} ^{1, \cdots, 1}\right) d t=-\frac{L \Gamma\left(q_{1}+q_{2}\right)}{q_{1}^{L+1}}+\frac{\Gamma\left(q_{1}+q_{2}\right) \varphi\left(0, q_{1}+q_{2}\right)}{q_{1}^{L}}$.

Note that Eq. (47) gives the required expression for the ergodic capacity at high SNRs in terms of simple elementary function. According to Eq. 477), the multiplexing gain is computed to be 1 and a smaller variance $\sigma_{z}^{2}$ leads to a higher ergodic capacity. Knowing the fact that the effect of pointing errors can be neglected when $A_{0} \rightarrow 1$ and $g \rightarrow \infty$ [14, then the approximate and the asymptotic expressions for the ergodic capacity of MIMO FSO systems in the absence of pointing errors are respectively evaluated as

$$
\begin{aligned}
C_{\mathrm{MIMO}, \mathrm{npe}} \approx & \frac{\log _{2}(\exp (1))}{\pi} \sum_{q=0}^{Q} \sum_{k=1}^{K} w_{k} \frac{\exp (-r L)(r L)^{q}}{q ! \Gamma(L+q)} \frac{L^{L} g^{L}}{2^{L}} \\
& 2^{q-1} G_{4,2}^{1,4}\left(\left.\frac{4 \frac{\gamma_{0}}{N_{r}}}{\left(\frac{(1+r)}{u_{k}} L\right)^{2}}\right|_{1,0} ^{\left.1,1, \frac{1-L-q}{2}, \frac{2-L-q}{2}\right)}\right)
\end{aligned}
$$


and

$$
\begin{aligned}
C_{\text {MIMO,npe,hsr }} \approx & \log _{2}(\exp (1))\left(2 \sum_{q=0}^{Q} \frac{\exp (-r L)(t L)^{q}}{q !} \varphi(0, L+q)\right. \\
& \left.-\sigma_{L}^{2}-2 \log (1+r)+\log \left(\frac{\gamma_{0}}{N_{r}}\right)\right)
\end{aligned}
$$

Note that Eq. 49 can be readily derived based on the definition of Mejier's G-function [27, Eq. (9.301)].

\subsection{Outage performance analysis}

Outage probability is also an important performance metric, which is defined as the probability that the instantaneous combined SNR $\gamma_{E G C}$ is lower than a given threshold $\gamma_{t h}$, that is

$P_{\text {out }}=\operatorname{Pr}\left(\gamma_{E G C}<\gamma\right)=\int_{0}^{\gamma_{\text {th }}} f_{S}(s) d s=\int_{0}^{\sqrt{\frac{N_{r} \gamma_{t h}}{\gamma_{0}}}} f_{S}(s) d s \approx \int_{0}^{\sqrt{\frac{N_{r} \gamma_{t h}}{\gamma_{0}}}} f_{\hat{S}}(s) d s$.

Substituting Eq. (24) into Eq. (51) and using Eq. 25), the outage performance for the MIMO FSO systems with pointing errors can be approximated as

$$
\begin{aligned}
P_{\text {out }, p e} \approx & \frac{1}{\sqrt{\pi}} \sum_{q=0}^{Q} \sum_{k=1}^{K} w_{k} \frac{\exp (-r L)(r L)^{q}}{q ! \Gamma(L+q)} \frac{L^{L} g^{L}}{2^{L}} \\
& G_{L+1, L+2}^{L+1,1}\left(\left.\frac{\sqrt{\frac{N_{r} \gamma_{t h}}{\gamma_{0}}}(1+r)}{u_{k} A_{0} F} L\right|_{L+q, L g / 2, \cdots, L g / 2,0} ^{1,1+L g / 2, \cdots, 1+L g / 2}\right) .
\end{aligned}
$$

Correspondingly, an approximate analytic expression for the outage performance of MIMO FSO systems without pointing errors can be readily carried out by

$$
\begin{aligned}
P_{\text {out }, \text { npe }} & \approx \frac{1}{\sqrt{\pi}} \sum_{q=0}^{Q} \sum_{k=1}^{K} w_{k} \frac{\exp (-r L)(r L)^{q}}{q ! \Gamma(L+q)} G_{1,2}^{1,1}\left(\left.\frac{\sqrt{\frac{N_{r} \gamma_{t h}}{\gamma_{0}}}(1+r)}{u_{k}} L\right|_{L+q, 0} ^{1}\right) \\
& \approx \frac{1}{\sqrt{\pi}} \sum_{q=0}^{Q} \sum_{k=1}^{K} w_{k} \frac{\exp (-r L)(r L)^{q}}{q ! \Gamma(L+q)} \gamma\left(L+q,\left(\frac{1+r}{u_{k}}\right) L \sqrt{\frac{N_{r} \gamma_{t h}}{\gamma_{0}}}\right)
\end{aligned}
$$


where $\gamma(\cdot, \cdot)$ denotes the lower incomplete gamma function and the second equality in Eq. 53 holds due to 43 .

\subsection{BER performance analysis}

For a OOK-based MIMO FSO systems, the average BER performance is given by [4]

$$
P_{b, p e}=\int_{0}^{\infty} \frac{1}{2} f(s) \operatorname{erfc}\left(\sqrt{\frac{\gamma_{0} s^{2}}{4 N_{r}}}\right) d s \approx \frac{1}{2 \sqrt{\pi}} \int_{0}^{\infty} f(\hat{s}) G_{1,2}^{2,0}\left[\left.\frac{\gamma_{0}}{4 N_{r}} \hat{s}^{2}\right|_{0,0.5} ^{1}\right] d \hat{s}
$$

where in Eq. (54), erfc (.) denotes the complementary error function [27, Eq. (8.250.4)], and we have expressed it in terms of Meijer's G-function [45, Eq. (8.4.14.1)]. Substituting Eq. (24) into Eq. (54) and using the formula [42, an approximate closed-form expression for the BER performance of MIMO FSO communication systems is obtained as

$$
\begin{aligned}
P_{b, p e} \approx & \frac{1}{\pi^{\frac{3}{2}}} \sum_{q=0}^{Q} \sum_{k=1}^{K} w_{k} \frac{\exp (-r L)(r L)^{q}}{q ! \Gamma(L+q)} \frac{L^{L} g^{L}}{2^{L}} 2^{q-2} \\
G_{L+3, L+2}^{2, L+2} & \left(\left.\frac{\gamma_{0}}{N_{r}\left(\frac{(1+r)}{u_{k} A_{0} F} L\right)^{2}}\right|_{0,0.5,\left(\frac{-\frac{L g}{2}}{2}\right)} ^{\frac{1-L-q}{2}, \frac{2-L-q}{2},\left(\frac{2-\frac{L g}{2}}{2}\right)^{\otimes L}, 1}\right)
\end{aligned}
$$

Correspondingly, an approximate analytic expression for the BER performance of MIMO FSO systems without pointing errors is obtained as

$P_{b, n p e} \approx \frac{1}{\pi^{\frac{3}{2}}} \sum_{q=0}^{Q} \sum_{k=1}^{K} w_{k} \frac{\exp (-r L)(r L)^{q}}{q ! \Gamma(L+q)} 2^{L} 2^{q-2} G_{3,2}^{2,2}\left(\left.\frac{\gamma_{0}}{N_{r}\left(\frac{(1+r)}{u_{k}} L\right)^{2}}\right|_{0,0.5} ^{\frac{1-L-q}{2}, \frac{2-L-q}{2}, 1}\right)$

\section{Numerical results and discussion}

In this section, we present some numerical results for the ergodic capacity, outage probability and BER performance of MIMO FSO systems over 
lognormal-Rician turbulence channels with pointing errors. The Monte Carlo simulation results are obtained through MATLAB, which are also included as a benchmark in all the figures. Note the pointing errors parameters have been used in most practical FSO systems [14, 46]. In all of the cases considered, we find that there is a good match between the analytically approximated and the simulated results. These results validate the accuracy of the derived expressions.

Fig. 5 depicts the simulated, approximate analytical, and high SNR ergodic capacity of MIMO FSO systems as a function of the average electrical SNR $\gamma_{0}$ for different apertures and pointing errors. Note that the pointing errors are considered here assuming values of normalized beam width and normalized jitter of $\left(w_{z} / R_{a}=5, \sigma_{z} / R_{a}=1\right)$, and $\left(w_{z} / R_{a}=10, \sigma_{z} / R_{a}=2\right)$. From the figure, we illustrate that the an approximate analytical expression Eq. 45 and an asymptotic expression Eq. 46 provide excellent agreement with the Monte Carlo simulation results, and thus verify the high accuracy of the proposed approximation. Also, it must be highlighted that the obtained analytical expression of ergodic capacity is very precise in the entire SNR regime, that is, overlaps with the simulation results from low to high SNR. As expected, the ergodic capacity is significantly increased as the apertures increase. For example, when $\left(w_{z} / R_{a}=10, \sigma_{z} / R_{a}=2\right)$ and $\gamma_{0}=50 \mathrm{~dB}$, the ergodic capacity for $M_{t}=1, N_{r}=2$ is $5.54 \mathrm{bits} / \mathrm{Hz} / \mathrm{s}$ while it is $7.68 \mathrm{bits} / \mathrm{Hz} / \mathrm{s}$ for $M_{t}=2, N_{r}=2$. In other words, the impacts of pointing errors can be mitigated greatly by utilizing the MIMO technique. Furthermore, the results presented in Table. 1 show that the obtained approximate expressions are also efficient for the MIMO FSO systems with larger apertures at both ends and the relative error decreases as SNR increases.

The impacts of turbulence conditions and apertures on the ergodic capacity of SIMO FSO systems are investigated in Fig. 6. By comparing these curves, we find the smaller variance $\sigma_{z}^{2}$ results in larger ergodic capacity. For example, when $M_{t}=1, N_{r}=2$, and $\gamma_{0}=50 \mathrm{~dB}$, the ergodic capacity for $\sigma_{z}^{2}=0.1$ is $9.41 \mathrm{bits} / \mathrm{Hz} / \mathrm{s}$ while it is $8.78 \mathrm{bits} / \mathrm{Hz} / \mathrm{s}$ for $\sigma_{z}^{2}=0.8$. As expected, the ergodic capacity can be improved with the increasing number of the apertures. 
In Fig. 7. we present a comparison between the proposed approximation and other methods for a SISO FSO link with pointing errors. Note that the Asymptotic1 results are derived from the Eq. (47) while the Asymptotic2 results are derived from the [23, Eq. (23)]. It is observed that the derived asymptotic results based on our method are closer to the theoretical results.

The outage performance of MIMO FSO systems over lognormal-Rician turbulence channels with pointing errors is shown in Fig. 8. As can be seen, the analytical expression in Eq. (52) provides an excellent match in the low-tomedium SNR regime while it acts as a lower bound at high SNRs. However, we note that it also can be regarded as a reasonable reference to the actual system performance since the simulation results lie within $0.3 \mathrm{~dB}$ in all cases examined. As expected, the outage performance is significantly improved as the apertures increase.

Fig. 9 depicts the BER performance of MIMO FSO systems for the considered channel model. It can be seen that the obtained approximate results overlap with the simulation results in the low-to-medium SNR regime while it acts as a lower bound at high SNR, and this is consistent with the results shown for outage performance. As expected, increasing the number of apertures improves the reliability of FSO systems.

\section{Conclusion}

In this paper, we have discussed the performance of MIMO FSO systems employing the EGC scheme over the lognormal-Rician turbulence channels with

pointing errors. A novel approximate analytical PDF expression for such a channel is developed and shown to be highly accurate over a wide range of channel conditions according to the results of KS goodness-of-fit tests results. Based on the proposed approximation, the approximate closed-form expressions for the ergodic capacity, outage probability, and BER performance of MIMO FSO systems with EGC diversity are formulated. The obtained approximate results for ergodic capacity are in excellent agreement with the Monte Carlo 


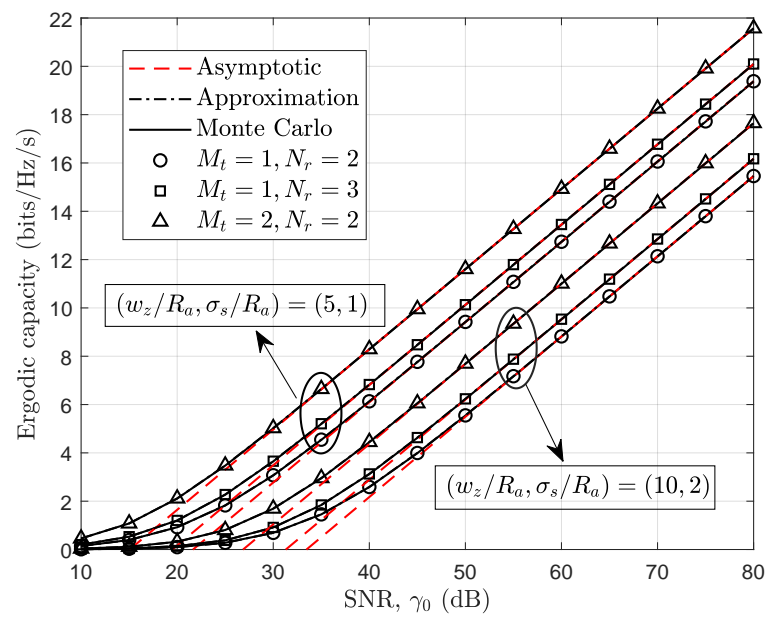

Figure 5: The ergodic capacity of MIMO FSO systems for different combinations of apertures and pointing errors when $r=4, \sigma_{z}^{2}=0.1$.

Table 1: The ergodic capacity of MIMO FSO systems for $M_{t}=3, N_{r}=3$, where relative error $\delta=\left|C_{\mathrm{MIMO}, \mathrm{pe}}-\hat{C}_{\mathrm{MIMO}, \mathrm{pe}}\right| / C_{\mathrm{MIMO}, \mathrm{pe}}$.

\begin{tabular}{|c|c|c|c|}
\hline $\mathrm{SNR}(\mathrm{dB})$ & $C_{\text {MIMO,pe }}(\mathrm{bits} / \mathrm{Hz} / \mathrm{s})$ & $\hat{C}_{\text {MIMO,pe }}(\mathrm{bits} / \mathrm{Hz} / \mathrm{s})$ & $\delta$ \\
\hline 0 & 0.1698 & 0.1661 & 0.0218 \\
\hline 10 & 1.1307 & 1.1129 & 0.0157 \\
\hline 20 & 3.6310 & 3.5869 & 0.0121 \\
\hline 30 & 6.8311 & 6.7535 & 0.0114 \\
\hline 40 & 10.1401 & 10.0273 & 0.0111 \\
\hline 50 & 13.4608 & 13.3126 & 0.0110 \\
\hline 60 & 16.7826 & 16.5991 & 0.0109 \\
\hline 70 & 20.1045 & 19.8857 & 0.0109 \\
\hline 80 & 23.4264 & 23.1723 & 0.0108 \\
\hline
\end{tabular}




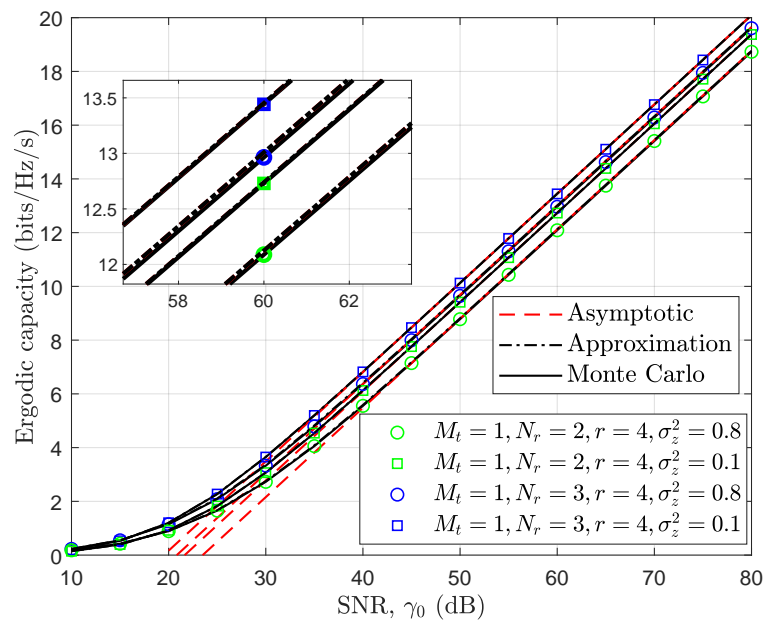

Figure 6: The ergodic capacity of SIMO FSO systems for different combinations of turbulence conditions and apertures when $w_{z} / R_{a}=5, \sigma_{s} / R_{a}=1$.

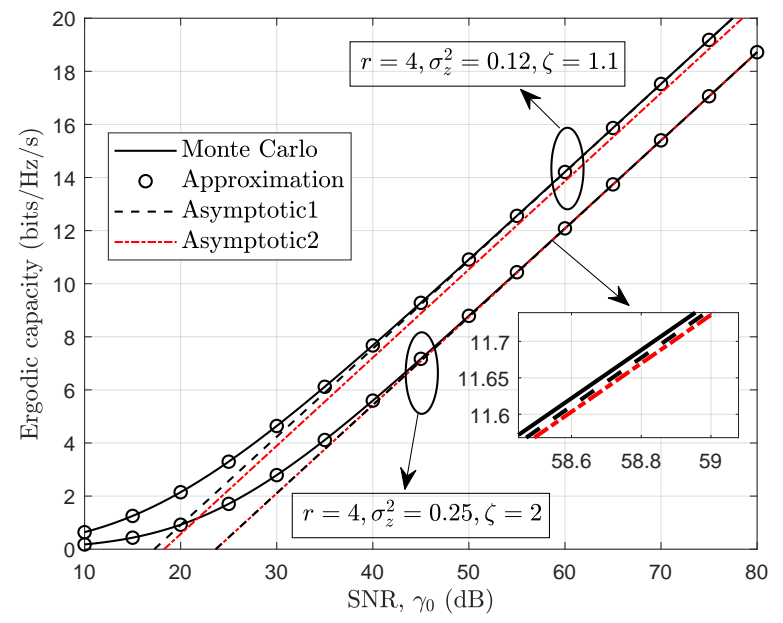

Figure 7: The comparison of asymptotic ergodic capacity for SISO FSO systems. 


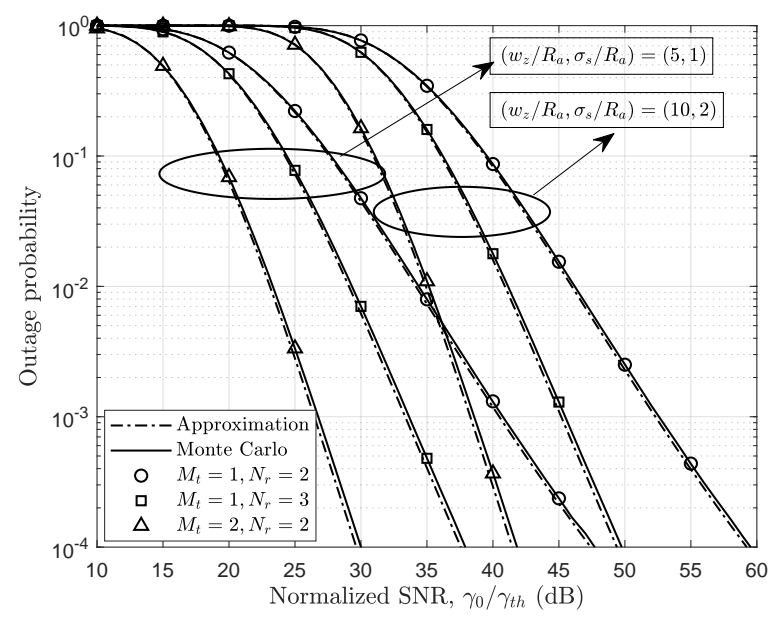

Figure 8: The outage probability of MIMO FSO systems for different combinations of apertures and pointing errors when $r=4, \sigma_{z}^{2}=0.1$.

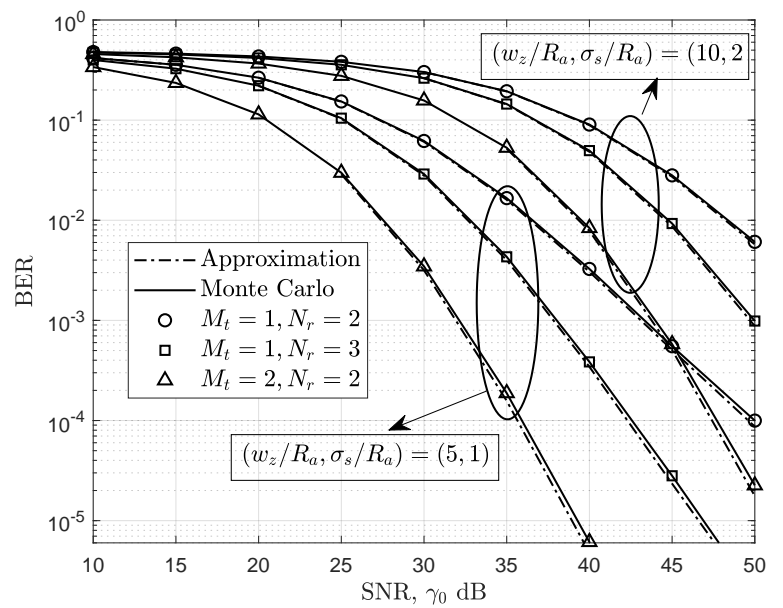

Figure 9: The BER performance of MIMO FSO systems for different combinations of apertures and pointing errors when $r=4, \sigma_{z}^{2}=0.1$. 
simulation results. Additionally, by comparing to the simulation results, the obtained results for outage probability and BER performance are highly efficient in the low-to-medium SNR regime while both of them act as a lower bound at high SNR.

Moreover, the results presented in this paper are based on the assumption of the i.i.d fading between transmitters and receivers. The independent but not necessarily identically distributed (i.i.n.d), and correlation cases will be considered in the near future.

\section{Appendix A}

To derive the Eq. (24), we mainly focus on solving the following integral:

$$
\int_{\frac{s}{F A_{0}}}^{\infty} \frac{1}{y} t_{1}^{L+q-1} \exp \left(-\frac{1+r}{u_{k}} t_{1} L\right) \log ^{L-1}\left(\frac{F A_{0} t_{1}}{s}\right)\left(\frac{s}{t_{1} F A_{0}}\right)^{\frac{L g}{2}} d t_{1} .
$$

Performing the change of variable $\frac{F A_{0} t_{1}}{s}=h$, Eq. (57) can be rewritten as

$$
\int_{1}^{\infty} \frac{1}{F A_{0}}\left(\frac{y}{F A_{0}}\right)^{L+q-1} h^{L+q-\frac{L g}{2}-1} \exp \left(-\frac{1+r}{u_{k}} \frac{s}{F A_{0}} L h\right) \log ^{L-1}(h) d h .
$$

Note that the integral involves in Eq. (58) can be evaluated as

$$
\begin{array}{r}
\int_{1}^{\infty} h^{L+q-\frac{L g}{2}-1} \exp \left(-\frac{1+r}{u_{k}} \frac{s}{F A_{0}} L h\right) \log ^{L-1}(h) d h=\Gamma(L)\left(\frac{1+r}{u_{k}} \frac{s}{F A_{0}} L\right)^{\frac{L g}{2}-(L+q)} \\
G_{L, L+1}^{L+1,0}\left(\left.\frac{s(1+r)}{u_{k} A_{0} F} L\right|_{L+q-\frac{L g}{2}, 0, \cdots, 0} ^{1, \cdots, 1}\right)
\end{array}
$$

where in Eq. (59), we have used the following integration formula [16, Eq. (79)]

$$
\int_{1}^{\infty} x^{v-1} \exp (-t x) \log ^{m}(x) d x=\Gamma(m+1) t^{-v} G_{m+1, m+2}^{m+2,0}\left(\left.t\right|_{v, 0, \cdots, 0} ^{1, \cdots, 1}\right) .
$$

Hence, combining Eqs. (21), 23), and (59) yields Eq. (24) after some algebraic manipulations. 


\section{Appendix B}

Since $\left\{z_{l}\right\}_{l=1}^{l=L},\left\{y_{l}\right\}_{l=1}^{l=L}$, and $\left\{u\left(r_{l}\right)\right\}_{l=1}^{l=L}$ are identically distributed variates with parameters $\left(\sigma_{z}^{2}\right),(r)$, and $\left(A_{0}, \xi\right)$; their first moments and variances are given by

$$
\begin{aligned}
\mathbb{E}\left[z_{l}\right] & =\mathbb{E}\left[y_{l}\right]=1 \\
\mathbb{E}\left[u\left(r_{l}\right)\right] & =\frac{A_{0} \xi^{2}}{1+\xi^{2}} \\
\mathbb{E}\left[z_{l}^{2}\right] & =\exp \left(\sigma_{z}^{2}\right) \\
\mathbb{E}\left[y_{l}^{2}\right] & =\frac{(2+r(4+r))}{(1+r)^{2}} \\
\mathbb{E}\left[u^{2}\left(r_{l}\right)\right] & =\frac{A_{0}^{2} \xi^{2}}{2+\xi^{2}} .
\end{aligned}
$$

The variance of $\epsilon_{1}$ and $\epsilon_{2}$ are equal to their second moments individually, which are respectively given by

$$
\mathbb{E}\left[\epsilon_{1}^{2}\right]=\frac{1}{L^{4}} \mathbb{E}\left[\left(\sum_{i=1}^{L-1} \sum_{j=i+1}^{L}\left(z_{i}-z_{j}\right)\left(y_{i}-y_{j}\right)\right)^{2}\right] \mathbb{E}\left[\left(\sum_{l=1}^{L} u\left(r_{l}\right)\right)^{2}\right]
$$

and

$$
\mathbb{E}\left[\epsilon_{2}^{2}\right]=\frac{1}{L^{2}} \mathbb{E}\left[\left(\sum_{i=1}^{L-1} \sum_{j=i+1}^{L}\left(z_{i} y_{i}-z_{j} y_{j}\right)\left(u\left(r_{i}\right)-u\left(r_{j}\right)\right)\right)^{2}\right] .
$$

Using the second and sixth equalities in Eq. (61), Eq. (62) is then transformed into

$\mathbb{E}\left[\epsilon_{1}^{2}\right]=\frac{1}{L^{3}} \mathbb{E}\left[\left(\sum_{i=1}^{L-1} \sum_{j=i+1}^{L}\left(z_{i}-z_{j}\right)\left(y_{i}-y_{j}\right)\right)^{2}\right]\left(\frac{A_{0}^{2} \xi^{2}}{2+\xi^{2}}+(L-1) \frac{A_{0}^{2} \xi^{4}}{\left(1+\xi^{2}\right)^{2}}\right)$.

Note that the computation for Eqs. 63 and 64 can be greatly simplified by using the Eqs. 65 and 66

$\mathbb{E}\left[\left(z_{i}-z_{j}\right)\left(z_{g}-z_{h}\right)\left(y_{i}-y_{j}\right)\left(y_{g}-y_{h}\right)\right]=\left\{\begin{array}{cc}\frac{(2+4 r)}{(1+r)^{2}}\left(2 \exp \left(\sigma_{z}^{2}\right)-2\right) & \text { if } i=g \text { and } j=h \\ 0 & \text { if } i \neq g \text { and } j \neq h \\ \frac{(1+2 r)}{(1+r)^{2}}\left(\exp \left(\sigma_{z}^{2}\right)-1\right) & \text { if } i=g \text { and } j \neq h\end{array}\right.$ 


$$
\begin{aligned}
& \mathbb{E}\left[\left(W_{i}-W_{j}\right)\left(u\left(r_{i}\right)-u\left(r_{j}\right)\right)\left(W_{g}-W_{h}\right)\left(u\left(r_{g}\right)-u\left(r_{h}\right)\right)\right]= \\
& \left\{\begin{array}{cl}
4\left(\frac{2+4 r+r^{2}}{(1+r)^{2}} \exp \left(\sigma_{z}^{2}\right)-1\right)\left(\frac{A_{0}^{2} \xi^{2}}{2+\xi^{2}}-\frac{A_{0}^{2} \xi^{4}}{\left(1+\xi^{2}\right)^{2}}\right) & \text { if } i=g \text { and } j=h \\
0 & \text { if } i \neq g \text { and } j \neq h \\
\left(\frac{\left(2+4 r+r^{2}\right)}{(1+r)^{2}} \exp \left(\sigma_{z}^{2}\right)-1\right)\left(\frac{A_{0}^{2} \xi^{2}}{2+\xi^{2}}-\frac{A_{0}^{2} \xi^{4}}{\left(1+\xi^{2}\right)^{2}}\right) & \text { if } i=g \text { and } j \neq h
\end{array} .\right.
\end{aligned}
$$

Hence, after some algebra, Eqs. (64) and (63) are simplified to Eqs. (38) and (39) respectively.

\section{Declaration of competing interest}

The authors declare that they have no known competing financial interests or personal relationships that could have appeared to influence the work reported in this paper.

\section{Acknowledgments}

The authors wish to thank team members of our research group for their kind help and valuable discussions in preparing the paper.

\section{References}

[1] M. Z. Chowdhury, M. T. Hossan, A. Islam, Y. M. Jang, A comparative survey of optical wireless technologies: Architectures and applications, IEEE Access 6 (2018) 9819-9840. doi:10.1109/ACCESS.2018.2792419.

[2] W. Gappmair, Further results on the capacity of free-space optical channels in turbulent atmosphere, IET Communications 5 (2011) 1262-1267(5).

[3] X. Zhu, J. Kahn, Free-space optical communication through atmospheric turbulence channels, IEEE Transactions on Communications 50 (8) (2002) 1293-1300. doi:10.1109/TCOMM.2002.800829. 
[4] Z. Wang, W.-D. Zhong, C. Yu, S. Fu, Performance improvement of onoff-keying free-space optical transmission systems by a co-propagating reference continuous wave light, Opt. Express 20 (8) (2012) 9284-9295. doi:10.1364/0E.20.009284

[5] A. L. C., Laser beam propagation through random media / Larry C. Andrews, Ronald L. Phillips, 2nd Edition, SPIE Press, Bellingham, Wash, 2005 .

[6] F. S. Vetelino, C. Young, L. Andrews, J. Recolons, Aperture averaging effects on the probability density of irradiance fluctuations in moderateto-strong turbulence, Appl. Opt. 46 (11) (2007) 2099-2108. doi:10.1364/ A0.46.002099.

[7] J. H. Churnside, S. F. Clifford, Log-normal rician probability-density function of optical scintillations in the turbulent atmosphere, J. Opt. Soc. Am. A 4 (10) (1987) 1923-1930. doi:10.1364/JOSAA.4.001923.

[8] F. Yang, J. Cheng, Coherent free-space optical communications in lognormal-Rician turbulence, IEEE Commun. Lett. 16 (11) (2012) 18721875. doi:10.1109/LCOMM. 2012.100812.121341.

[9] A. Jurado-Navas, J. M. Garrido-Balsells, J. F. Paris, A. Puerta-Notario, A unifying statistical model for atmospheric optical scintillation (2011). arXiv:1102.1915.

[10] N. Wang, J. Cheng, Moment-based estimation for the shape parameters of the gamma-gamma atmospheric turbulence model., Opt. Express 18 (12) (2010) 12824-12831. doi:10.1364/0E.18.012824.

[11] I. S. Ansari, F. Yilmaz, M.-S. Alouini, Performance analysis of free-space optical links over malaga turbulence channels with pointing errors, IEEE Transactions on Wireless Communications 15 (1) (2016) 91-102. doi:10. 1109/TWC.2015.2467386. 
[12] D. K. Borah, D. G. Voelz, Pointing error effects on free-space optical communication links in the presence of atmospheric turbulence, Journal of Lightwave Technology 27 (18) (2009) 3965-3973. doi:10.1109/JLT.2009. 2022771 .

[13] A. A. Farid, S. Hranilovic, Outage capacity optimization for free-space optical links with pointing errors, Journal of Lightwave Technology 25 (7) (2007) 1702-1710. doi:10.1109/JLT.2007.899174.

[14] R. Boluda-Ruiz, A. García-Zambrana, B. Castillo-Vázquez, C. CastilloVázquez, On the capacity of MISO FSO systems over Gamma-Gamma and misalignment fading channels, Opt. Express 23 (17) (2015) 22371-22385. doi:10.1364/OE.23.022371

[15] M. R. Bhatnagar, Z. Ghassemlooy, Performance analysis of gamma-gamma fading FSO MIMO links with pointing errors, Journal of Lightwave Technology 34 (9) (2016) 2158-2169. doi:10.1109/JLT.2016.2526053.

[16] M. Miao, X. Li, Novel approximate distribution of the sum of gammagamma variates with pointing errors and applications in MIMO FSO links, Optics Communications 486 (2021) 126780. doi:https://doi.org/10. 1016/j.optcom.2021.126780

[17] J. Ding, S. Yu, Y. Fu, J. Ma, L. Tan, New approximate and asymptotic closed-form expressions for the outage probability and the average ber of MIMO-FSO system with MRC diversity technique over GammaGamma fading channels with generalized pointing errors, Optics Communications 456 (2020) 124633. doi:https://doi.org/10.1016/j.optcom. 2019.124633

[18] On the distribution of the sum of málaga- $\mathcal{M}$ random variables and applications.

[19] M. J. Saber, S. M. S. Sadough, On secure free-space optical communications 
over Malaga Turbulence Channels, IEEE Wireless Communications Letters 6 (2) (2017) 274-277. doi:10.1109/LWC.2017.2671872.

[20] M. Miao, X. Li, Parameter estimation of the lognormal-rician channel model using saddlepoint approximation, IEEE Access 8 (2020) 152924152931. doi:10.1109/ACCESS. 2020.3016683.

[21] M. Miao, X. Li, Error performance analysis of optical communication over lognormal-Rician turbulence channel using Gram-Charlier Series, Journal of Optical Communications 0 (2021).

[22] F. Yilmaz, M.-S. Alouini, Novel asymptotic results on the high-order statistics of the channel capacity over generalized fading channels, in: 2012 IEEE 13th International Workshop on Signal Processing Advances in Wireless Communications (SPAWC), 2012, pp. 389-393. doi:10.1109/SPAWC. 2012.6292934

[23] I. S. Ansari, M.-S. Alouini, J. Cheng, On the capacity of FSO Links under Lognormal and Rician-Lognormal Turbulences, in: 2014 IEEE 80th Vehicular Technology Conference (VTC2014-Fall), 2014, pp. 1-6. doi: 10.1109/VTCFall.2014.6966082.

[24] S. M. Navidpour, M. Uysal, M. Kavehrad, BER performance of free-space optical transmission with spatial diversity, IEEE Transactions on Wireless Communications 6 (8) (2007) 2813-2819. doi:10.1109/TWC.2007.06109.

[25] P. Deng, M. Kavehrad, Z. Liu, Z. Zhou, X. Yuan, Capacity of MIMO free space optical communications using multiple partially coherent beams propagation through non-Kolmogorov strong turbulence, Opt. Express 21 (13) (2013) 15213-15229. doi:10.1364/OE.21.015213.

[26] V. P. Thanh, C.-T. Truong, T. P. Anh, On the MGF-based approximation of the sum of independent gamma-gamma random variables, in: Proc. 2015 IEEE 81st Vehicular Technology Conference (VTC Spring), 2015, pp. 1-5. doi:10.1109/VTCSpring.2015.7145970, 
[27] I. S. Gradshteyn, I. M. Ryzhik, Table of integrals, series, and products, seventh Edition, Elsevier/Academic Press, Amsterdam, 2007, translated from the Russian, Translation edited and with a preface by Alan Jeffrey and Daniel Zwillinger, With one CD-ROM (Windows, Macintosh and UNIX).

[28] X. Liu, Free-space optics optimization models for building sway and atmospheric interference using variable wavelength, IEEE Transactions on Communications 57 (2) (2009) 492-498. doi:10.1109/TCOMM. 2009.02.070089

[29] E. Farooq, A. Sahu, S. K. Gupta, Survey on FSO communication systemlimitations and enhancement techniques, in: V. Janyani, M. Tiwari, G. Singh, P. Minzioni (Eds.), Optical and Wireless Technologies, Springer Singapore, Singapore, 2018, pp. 255-264.

[30] N. D. Chatzidiamantis, G. K. Karagiannidis, On the distribution of the sum of gamma-gamma variates and applications in RF and Optical Wireless Communications, IEEE Transactions on Communications 59 (5) (2011) 1298-1308. doi:10.1109/TCOMM.2011.020811.090205.

[31] L. Fenton, The sum of log-normal probability distributions in scatter transmission systems, IRE Transactions on Communications Systems 8 (1) (1960) 57-67. doi:10.1109/TCOM.1960.1097606.

[32] F. Yilmaz, M.-S. Alouini, A novel unified expression for the capacity and bit error probability of wireless communication systems over generalized fading channels, IEEE Transactions on Communications 60 (7) (2012) 1862-1876. doi:10.1109/TCOMM.2012.062512.110846

[33] C. Fox, The $\mathrm{G}$ and $\mathrm{H}$ functions as symmetrical fourier kernels, Transactions of the American Mathematical Society 98 (3) (1961) 395-429.

[34] M. Abramowitz, I. A. Stegun (Eds.), Handbook of Mathematical Functions with Formulas, Graphs, and Mathematical Tables, tenth printing Edition, U.S. Government Printing Office, Washington, DC, USA, 1972. 
[35] J. Wu, N. Mehta, J. Zhang, Flexible lognormal sum approximation method, in: GLOBECOM '05. IEEE Global Telecommunications Conference, 2005., Vol. 6, 2005, pp. 3413-3417. doi:10.1109/GLOCOM.2005.1578407.

[36] B. Kumbhani, R. S. Kshetrimayum, MIMO Wireless Communications over Generalized Fading Channels, 1st Edition, CRC Press, Inc., USA, 2017.

[37] M. Yacoub, The $\kappa-\mu$ distribution and the $\alpha-\mu$ distribution, IEEE Antennas Propag. Mag. 49 (2007) 68-81.

[38] B. D. Carter, M. D. Springer, The distribution of products, quotients and powers of independent $\mathrm{H}$-function variates, SIAM Journal on Applied Mathematics 33 (4) (1977) 542-558.

[39] A. M. Mathai, The H-function theory and applications, Springer, New York, 2009.

[40] V. S. Adamchik, O. I. Marichev, The algorithm for calculating integrals of hypergeometric type functions and its realization in reduce system, in: Proceedings of the International Symposium on Symbolic and Algebraic Computation, ISSAC '90, Association for Computing Machinery, New York, NY, USA, 1990, pp. 212-224. doi:10.1145/96877.96930.

[41] A. Papoulis, S. U. Pillai, Probability, Random Variables, and Stochastic Processes, 4th Edition, McGraw Hill, Boston, 2002.

[42] Wolfram Research, Inc., The Wolfram functions site, https://functions. wolfram.com/HypergeometricFunctions/MeijerG/21/02/03/01/0002/.

[43] Wolfram Research, Inc., The Wolfram functions site, https://functions. wolfram.com/HypergeometricFunctions/MeijerG/03/01/03/12/0004

[44] E. Bayaki, R. Schober, R. K. Mallik, Performance analysis of MIMO freespace optical systems in gamma-gamma fading, IEEE Transactions on Communications 57 (11) (2009) 3415-3424. doi:10.1109/TCOMM.2009. 11.080168 . 
[45] A. P. Prudnikov, Bryčkov, Yu A. and Maričev, O. I., Integrals and Series of Special Functions, Science, RUS, 1983.

[46] R. Boluda-Ruiz, A. García-Zambrana, B. Castillo-Vázquez, C. CastilloVázquez, Impact of nonzero boresight pointing error on ergodic capacity of MIMO FSO communication systems, Opt. Express 24 (4) (2016) 35133534. doi:10.1364/0E.24.003513. 